\title{
Representación del conocimiento y percepción subjetiva del proceso de aprendizaje profesional: estudio cualitativo en personal de enfermería
}

\author{
M. Jiménez-Adán a , L.M. Casas-García b , R. Luengo-González ${ }^{\text {b }}$
}

\begin{abstract}
Objetivo. Presentamos una investigación cualitativa cuyo objetivo fue conocer las percepciones de un grupo de enfermeras y enfermeros sobre su proceso de aprendizaje profesional. Materiales y métodos. Se utilizó una metodología de análisis de textos que combinó el análisis de contingencias y las Redes Asociativas Pathfinder. Resultados y conclusiones. Los resultados indican que el factor más importante del aprendizaje profesional es la relación personal y la ayuda de otros compañeros, no sólo durante la práctica profesional, sino también durante la formación inicial.
\end{abstract}

Palabras clave. Aprendizaje profesional. Enfermería. Investigación cualitativa. Representación del conocimiento.

Knowledge representation and perception of the professional learning process: qualitative study in nursing

Aim. We present a qualitative research whose goal was to determine the perceptions of a group of nurses about their professional learning process. Materials and methods. We used a text analysis methodology that combined contingency analysis and Pathfinder Associative Networks. Results and conclusions. The results indicate that the most important thing in professional learning is the personal relationship and the help of other colleagues, not only during practice, but also during initial training.

Key words. Knowledge representation. Nursing. Professional education. Qualitative research.

\section{Introducción}

La formación en enfermería, de forma similar a lo que ocurre en otras profesiones, tiene dos etapas: la formación inicial durante la carrera y la formación continuada en el ejercicio de la profesión.

Si bien la formación durante la carrera está académicamente organizada y ha sido objeto de amplios estudios, la formación durante la práctica profesional depende, en su gran mayoría, de la colaboración con otros compañeros y se convierte, en muchos casos, en un aprendizaje de tipo artesanal, del que son responsables profesionales que están asumiendo una función, la docente, para la cual no se han preparado.

El primer objetivo de este trabajo es conocer cuáles son las percepciones, e incluso los sentimientos que el personal de enfermería tiene acerca de su propia formación, tanto de la recibida durante su formación inicial como en la práctica profesional.

El problema de investigación planteado entra, pues, dentro del campo de la representación del conocimiento, en el que se hace uso de técnicas como las Redes Asociativas Pathfinder [1], basadas en la puntuación de la similaridad entre conceptos que, de forma automatizada, obtienen y muestran de forma gráfica el contenido y estructura del conocimiento de las personas.

El segundo objetivo de nuestro trabajo consiste en explorar métodos de investigación que nos ayuden en la obtención de datos y representación del conocimiento, como hemos señalado, accediendo a los conocimientos y significados más profundos de las personas.

La investigación sobre la formación del personal de enfermería ha empleado distintos enfoques
Hospital Universitario Infanta Cristina.

b Facultad de Educación. Universidad de Extremadura. Badajoz, España.

Correspondencia Dr. Luis M. Casas García. Facultad de Educación. Universidad de Extremadura. Campus Universitario. Avda. Elvas, s/n. E-06071 Badajoz

E-mail

luisma@unex.es 
metodológicos, de tipo cuantitativo y, sobre todo desde la década de los noventa, cualitativo [2-4].

En un trabajo como el que planteamos, en el que interesa explorar y llegar a conocer cuáles son las percepciones de un grupo de personas, consideramos que una aproximación desde la metodología cualitativa, y particularmente desde el enfoque de la fenomenología, resulta el más adecuado.

En este enfoque, más que en el estudio del grupo en su conjunto, se hace especial énfasis en los individuos y en su experiencia subjetiva, mediante el análisis de contenido de las descripciones que hacen sobre sus vivencias a través del lenguaje hablado o del texto. En esa línea se han publicado otros trabajos [5-9].

Las técnicas más utilizadas para la recogida de datos desde este enfoque han sido la entrevista cualitativa, la observación participante y las técnicas narrativas.

La entrevista cualitativa proporciona datos de gran riqueza, pues suele hacerse de forma individual, pero resulta, por ello mismo, una técnica muy laboriosa y que no suele implicar a muchos sujetos de investigación.

La técnica de observación participante se concreta la mayor parte de las veces en forma de grupos de discusión en los que se trata una cuestión, planteada por el investigador, que recoge y analiza las intervenciones [10,11]. Esta técnica, sin embargo, tiene una limitación, y es el hecho de que, en situaciones de interacción social, los sujetos pueden tender a distorsionar sus convicciones y manifestar opiniones acordes con lo que se considera correcto (fenómeno conocido como 'deseabilidad social').

Las técnicas narrativas incluyen una gran variedad de prácticas como autobiografías, historias de vida o historias profesionales, que cuando incluyen narraciones sobre las carreras profesionales de los individuos, se pueden utilizar para valorar la repercusión de la formación y las experiencias vitales en sus roles y actitudes. Dado que el sujeto cumplimenta individualmente la información que se le pide, se aminora el efecto de deseabilidad social antes señalado.

Para concretar cuál es el estado de la cuestión en cuanto al tema de investigación y en cuanto a la utilización de las metodologías antes citadas, hemos llevado a cabo una amplia revisión bibliográfica, que ha incluido las siguientes bases de datos:
- Bases documentales extranjeras: base ERIC.

- Bases documentales españolas: base de datos de tesis doctorales Teseo, base de datos de enfermería Cuiden, base de datos de enfermería Cuidatge, base de datos de bibliografía general Compludoc.

- Bases documentales de revistas en español: base de datos de Revista Española de Salud Pública, base de datos de la revista Rol de Enfermería.

Los descriptores utilizados para la búsqueda han sido los correspondientes a 'educación", 'formación', 'enfermería, 'investigación en enfermería', 'investigación cualitativa' y 'representación del conocimiento', así como combinaciones de dichos descriptores.

Aunque por la limitada extensión de este trabajo no describiremos detalladamente el resultado de la búsqueda, podemos concluir que existen dos líneas claramente definidas. Por una parte encontramos estudios en los que la relación de metodología cualitativa con enfermería se debe únicamente a que se utiliza a los alumnos de cursos de enfermería para llevar a cabo estudios sobre la aplicación de la metodología cualitativa en la formación en general. Por otra, hay trabajos en los que se aplica la metodología cualitativa a estudios sobre salud, aunque en algunos $[2,3,7,8,12]$ se utiliza también para la obtención de datos acerca de las percepciones de los estudiantes sobre su propia formación y particularmente sobre la importancia que le conceden a las prácticas.

No hemos encontrado estudios en los que se emplee la metodología cualitativa cuando se trata, como en nuestro caso, de estudiar la percepción sobre la formación durante la carrera y durante la práctica profesional que tienen los profesionales de enfermería ya en activo.

Consideramos que la combinación de técnicas narrativas con las técnicas de representación de conocimiento a que antes hemos hecho referencia, puede ofrecernos una información muy rica acerca del modo en que enfermeros y enfermeras en activo entienden su proceso de formación.

\section{Materiales y métodos}

Para la selección de los participantes en el estudio se utilizó un procedimiento de recogida de datos en 'bola de nieve', mediante el cual se pidió cola- 
Tabla. Categorías de análisis de los textos.

\begin{tabular}{|c|c|c|}
\hline Aprendizaje en la carrera ${ }^{a}$ & Aprendizaje profesional ${ }^{\mathrm{b}}$ & Percepciones y sentimientos ${ }^{c}$ \\
\hline 1. Enseñanza teórica buena & 12. Enseñanza científica & 24. Desmotivación en los alumnos \\
\hline 2. Enseñanza teórica mala & 13. Enseñanza artesanal & 25. Desilusión \\
\hline 3. Teórica por enfermeros & 14. Enseñanza por enfermeros & $\begin{array}{l}\text { 26. Falta de preparación } \\
\text { para enseñar }\end{array}$ \\
\hline 4. Teórica por médicos & $\begin{array}{l}\text { 15. Enfermeros no quieren } \\
\text { enseñar a los compañeros }\end{array}$ & $\begin{array}{l}\text { 27. Agradecimiento } \\
\text { al compañerismo }\end{array}$ \\
\hline 5. Prácticas buenas & 16. Enseñanza rutinaria & 28. Vocación \\
\hline 6. Prácticas malas & 17. Aprendizaje por cuenta propia & 29. Autoconfianza \\
\hline 7. Prácticas por enfermeros & 18. Aprendizaje comienza en el trabajo & 30. Satisfacción \\
\hline 8. Prácticas por monitores & 19. Problema por la gran especialización & 31. Temor por la responsabilidad \\
\hline $\begin{array}{l}\text { 9. Enfermeros no quieren } \\
\text { enseñar a alumnos }\end{array}$ & $\begin{array}{l}\text { 20. No perfeccionamiento } \\
\text { profesional observado }\end{array}$ & 32. Interés por aprender \\
\hline $\begin{array}{l}\text { 10. Necesidad de enfermeros } \\
\text { docentes en las prácticas }\end{array}$ & $\begin{array}{l}\text { 21. Necesidad de enfermeros } \\
\text { docentes en el trabajo }\end{array}$ & 33. Capacidad para enseñar \\
\hline $\begin{array}{l}\text { 11. Necesidad de más prácticas } \\
\text { en la carrera }\end{array}$ & 22. Necesidad de formación continuada & $\begin{array}{l}\text { 34. Aspectos personales } \\
\text { en el trato a enfermos }\end{array}$ \\
\hline \multicolumn{3}{|c|}{ 23. Aprendizaje en el trabajo por médicos } \\
\hline $\begin{array}{l}\text { a Expresiones relacionadas con la form } \\
\text { sido su aprendizaje en los diferentes s } \\
\text { realidad profesional. }\end{array}$ & $\begin{array}{l}\text { rendieron durante su época como estudiant } \\
\text { de han ejercido; ' }{ }^{\circ} \text { Expresiones que reflejen s }\end{array}$ & $\begin{array}{l}\text { bxpresiones que se refieren a cómo ha } \\
\text { timientos y percepciones en cuanto a su }\end{array}$ \\
\hline
\end{tabular}

boración a determinadas personas que, a su vez, la solicitaron a otras. La muestra final estuvo formada por 13 enfermeros y 26 enfermeras de los servicios de Urgencias, Quirófano y Neurología del Hospital Infanta Cristina de Badajoz. La media de edad fue de 42,8 años (rango: 24-60 años). En cuanto a los años de experiencia profesional, la media fue de 19,33 años (rango: 1-37 años).

Mediante este procedimiento se obtuvieron datos de aquellos informantes voluntarios que mostraron más interés en contestar, por lo que es de suponer que ofrecieron una mayor riqueza en sus datos.

Para la recogida de información se pidió a los participantes que redactaran un texto en el que indicaran cómo habían aprendido la profesión de enfermería, cuáles habían sido sus experiencias y qué les había parecido.

Para el análisis del contenido de los textos recogidos se utilizó el análisis de contingencias [13]. En esta técnica se parte del postulado de que dos elementos asociados en un escrito lo estarán del mismo modo en el pensamiento del sujeto, y a mayor número de documentos en que dos conceptos aparezcan asociados, mayor proximidad existirá entre tales conceptos.

Tras una primera revisión de los textos, se establecieron unas categorías de análisis (representadas por frases relativas a un determinado concepto), encuadradas en dimensiones (Tabla).

A continuación se analizó qué categorías aparecían de forma concurrente en un mismo do- 


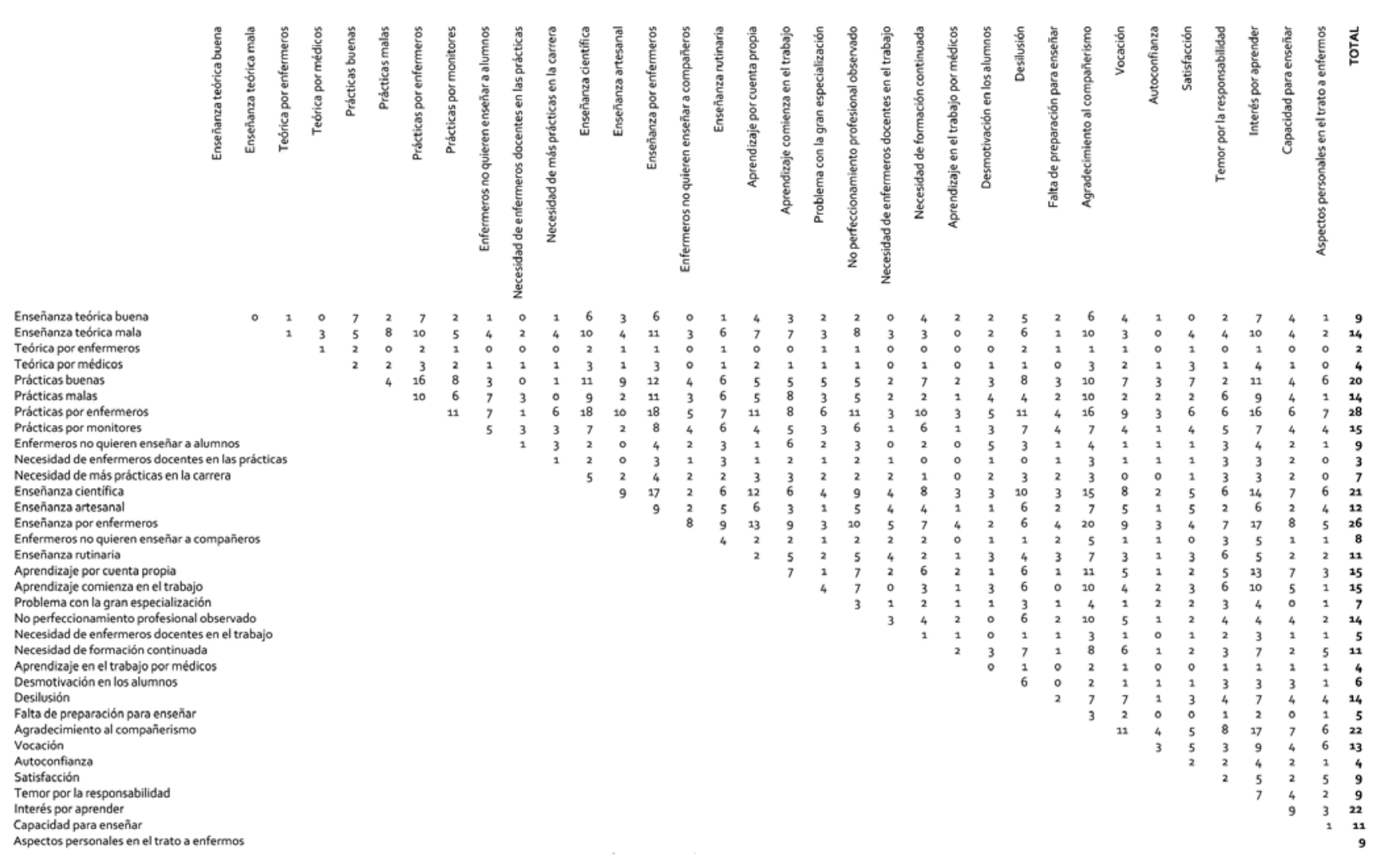

Figura 1. Matriz de contingencias.
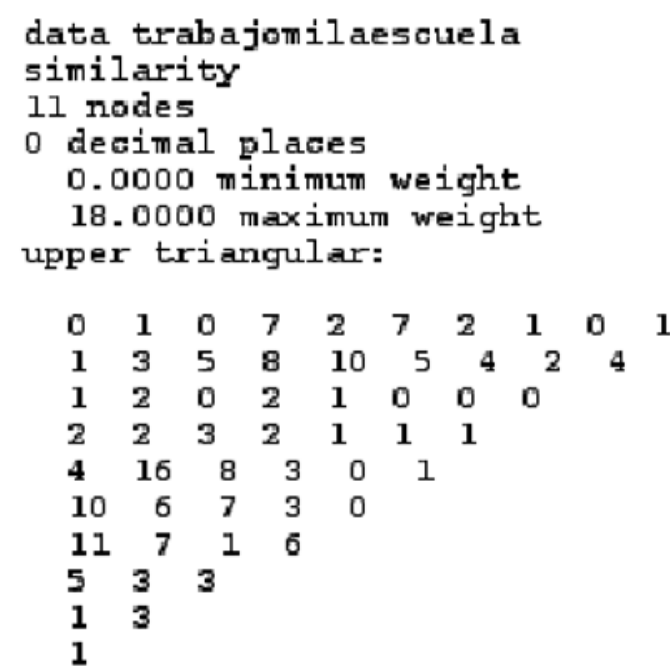

Figura 2. Fichero del programa KNOT. cumento y en cuántos documentos de distintas personas se daban dichas concurrencias.

Los datos obtenidos se recogieron en una matriz de contingencias en la que dos categorías se relacionarían más en la medida en que aparecieran a la misma vez en mayor número de documentos.

Esta relación se puede interpretar como una medida de proximidad y representarse gráficamente mediante Redes Asociativas Pathfinder que, utilizando el software KNOT [14] y de forma automatizada, se pueden emplear para representar el conocimiento en distintos campos $[15,16]$.

\section{Resultados}

A partir del análisis de los textos producidos por los participantes, se elaboró una matriz de contingencias (Fig. 1). 


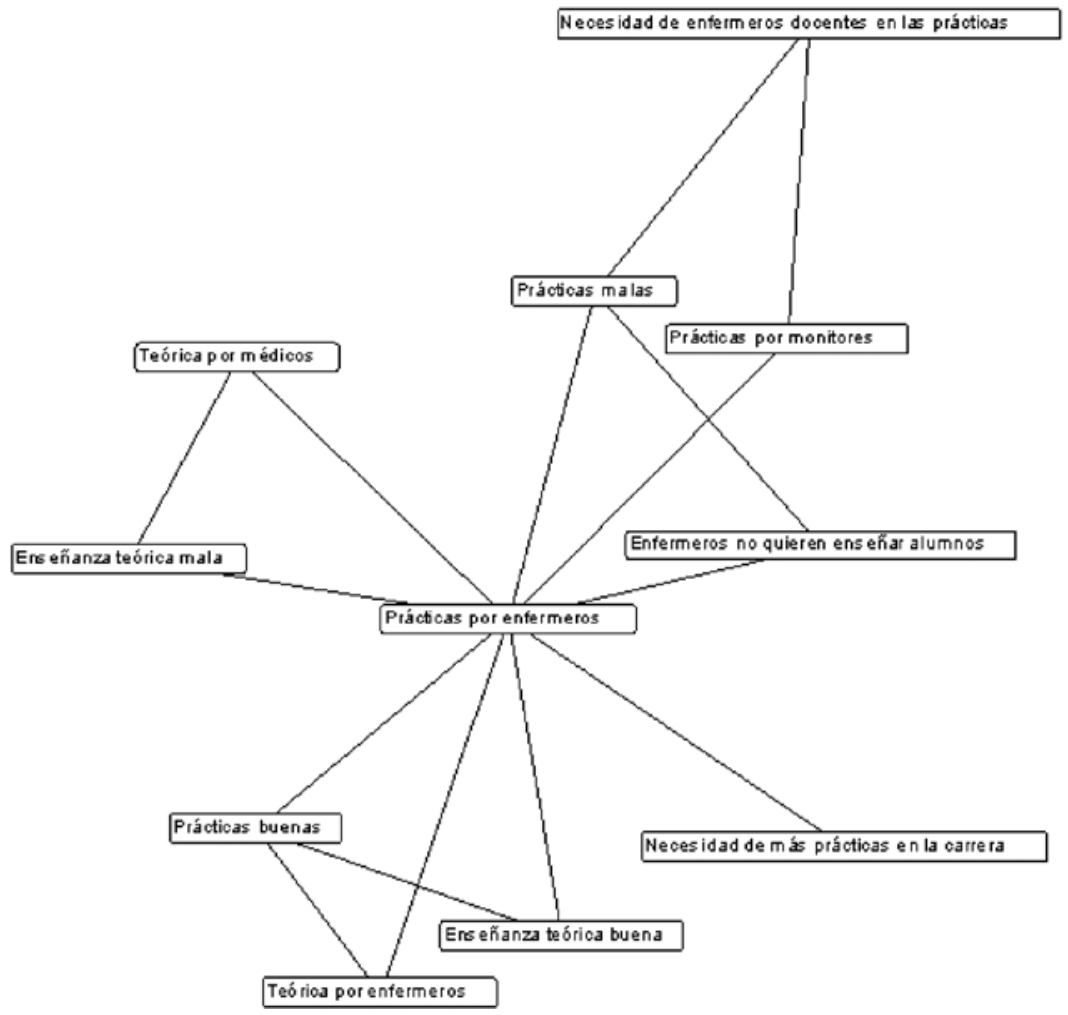

Figura 3. Red Asociativa Pathfinder: dimensión 'aprendizaje en la carrera'.

El programa KNOT, antes mencionado, utiliza como entrada de datos ficheros de texto con unas determinadas características (Fig. 2).

En nuestro caso, los datos fueron los tomados de la matriz de contingencias y el proceso se repitió para las tres dimensiones encontradas. A partir de estos ficheros, se obtuvieron las Redes Asociativas Pathfinder, representaciones gráficas de la relación entre categorías (Figs. 3, 4, 5 y 6).

En la figura 3 observamos cómo la categoría central es 'prácticas por enfermeros', lo cual interpretamos en el sentido de que, durante la carrera, fue la principal fuente de aprendizaje.

Encontramos una relación entre 'prácticas por enfermeros', 'prácticas buenas' 'teórica por enfermeros' y 'enseñanza teórica buena', lo que, en nuestra opinión, indica que hay un reconocimiento de que las prácticas, impartidas por profesionales de enfermería no docentes, son apreciadas como buenas y se complementan con una enseñanza teórica buena. Sin embargo, aparece una relación entre 'enseñanza teórica mala' y 'enseñanza teórica por médicos', que indica que no es $\tan$ valorada en este caso.

En cuanto a la red presentada en la figura 4, destacan, por una parte, la categoría 'enseñanza por enfermeros', y por otra, 'enseñanza científica', lo que podemos interpretar como que éstas son las preocupaciones principales de las personas que han participado en el trabajo y que asocian ambas categorías.

En la figura 5 observamos que la categoría central es 'agradecimiento al compañerismo', relacionada con otras, como 'satisfacción', 'vocación' o 'interés por aprender' y con 'aspectos personales en el trato a enfermos', sentimientos muy relacionados con la profesión de enfermería.

Si observamos la relación entre todas las categorías, en la figura 6, las más destacadas son la número 7 ('práctica por enfermeros') y la número 14 ('enseñanza por enfermeros'), lo que denota su importancia.

Destaca también la relación con la número 10 ('necesidad de enfermeros docentes durante las 


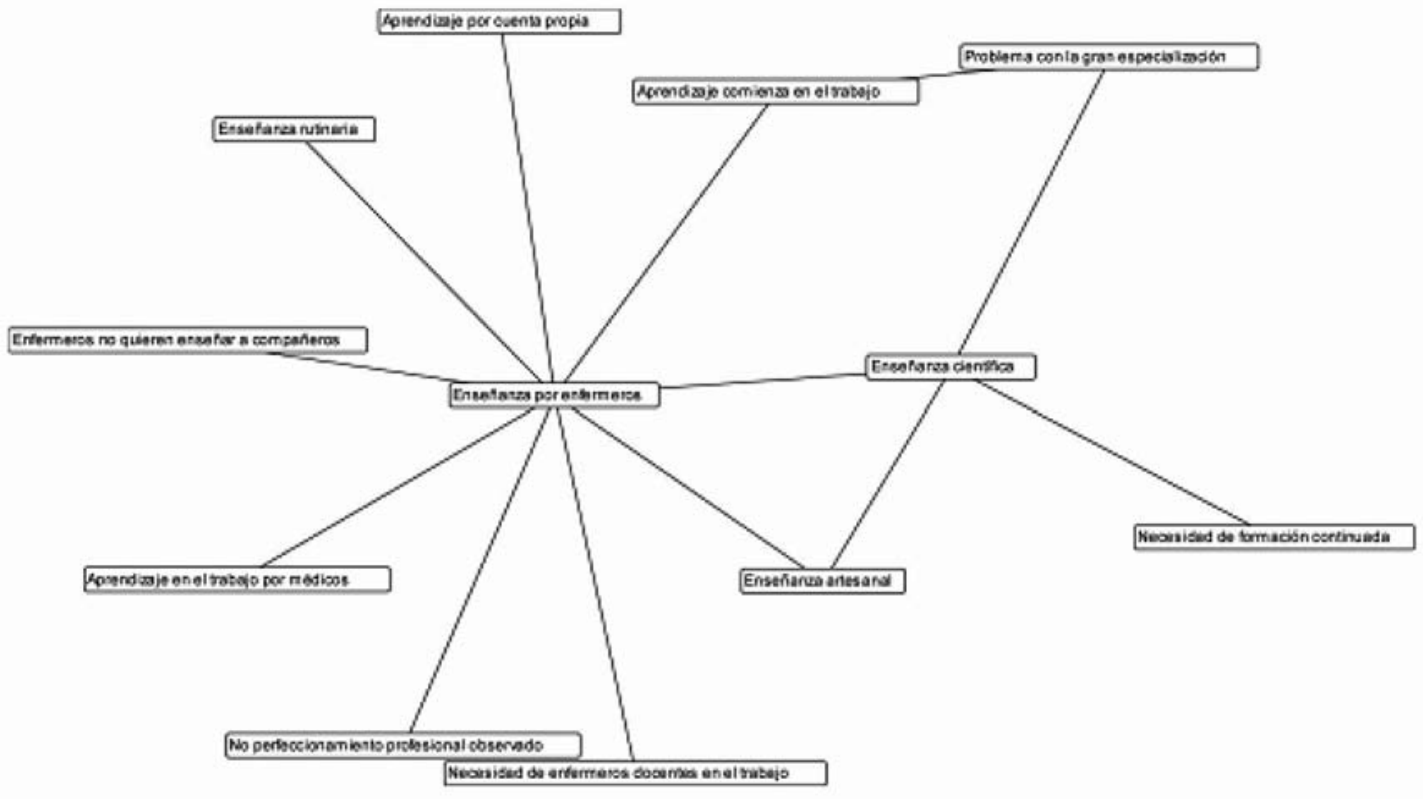

Figura 4. Red Asociativa Pathfinder: dimensión 'aprendizaje en práctica profesional'.

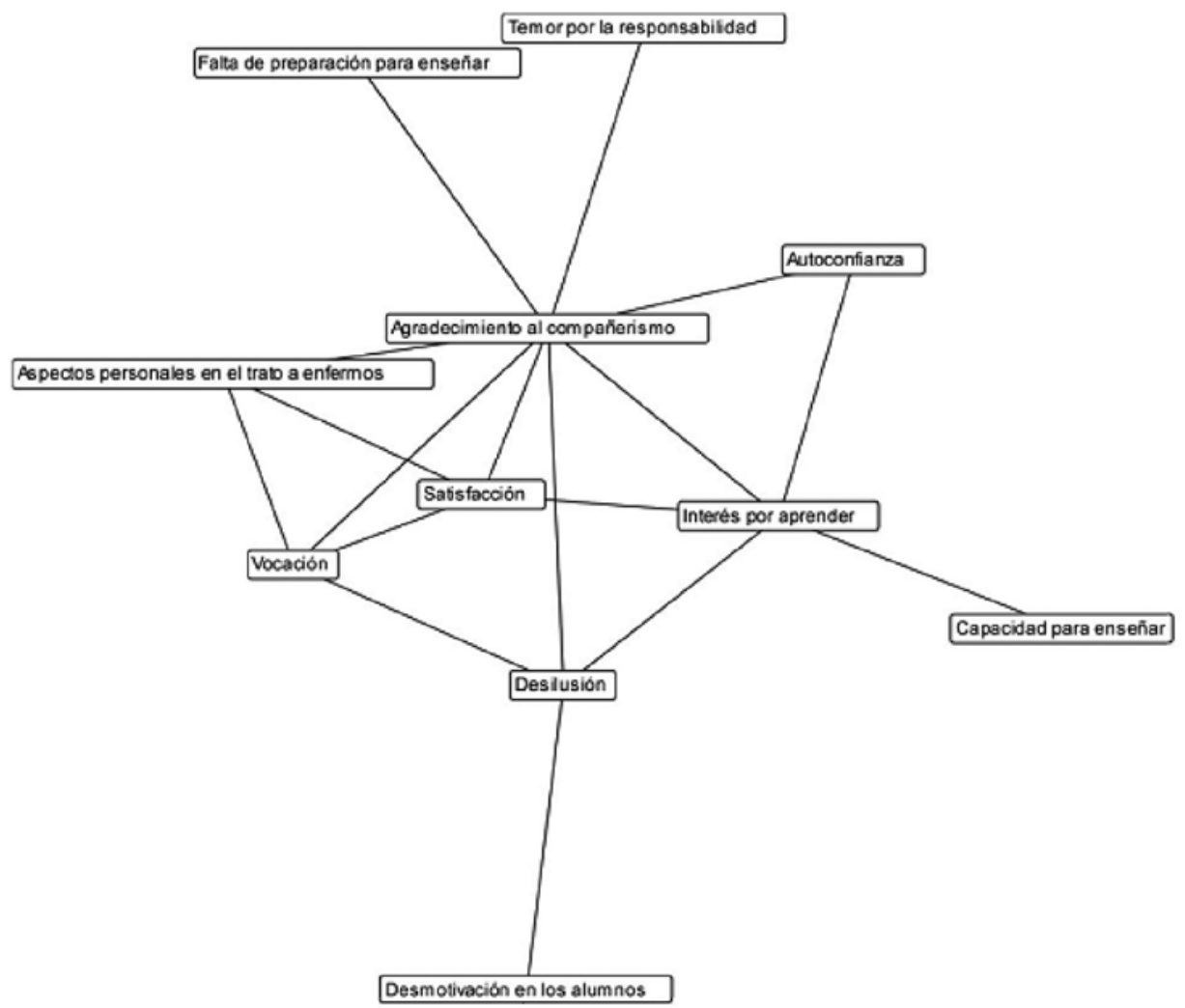

Figura 5. Red Asociativa Pathfinder: dimensión 'percepciones y sentimientos'. 


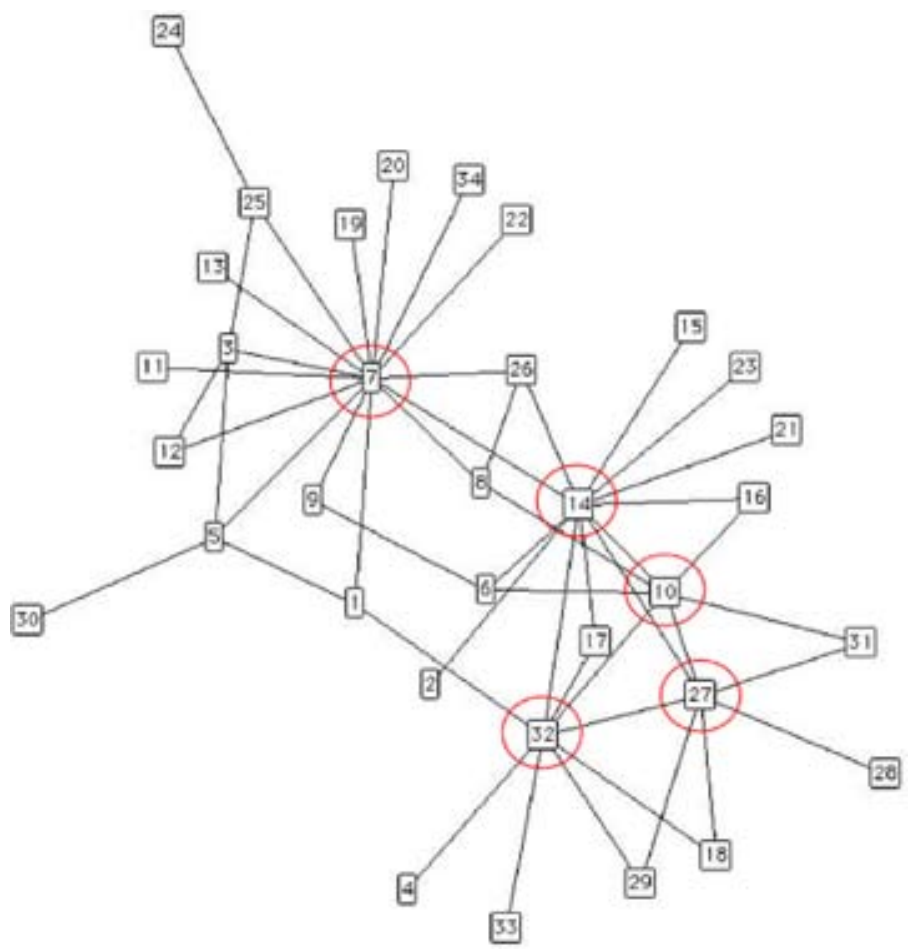

Figura 6. Red Asociativa Pathfinder: todas las dimensiones.

prácticas'), con la número 27 ('agradecimiento al compañerismo') y con la número 32 ('interés por aprender').

\section{Discusión}

No podemos comparar los resultados obtenidos en nuestro estudio con otros, pues no hemos encontrado referencias de trabajos similares que utilicen la misma metodología (combinación de análisis de contenido con técnicas de representación del conocimiento), ni que estén realizados con profesionales en activo.

Como limitaciones, podemos mencionar la muestra empleada. Aunque es suficiente, pues está en torno al tamaño habitual en estudios cualitativos y está constituida por buenos informantes, podría mejorarse si fuera más representativa de la población completa.

Consideramos además que sería necesario un estudio más en profundidad sobre el tipo de relaciones que se establecen entre las distintas cate- gorías, lo que requeriría un análisis más completo, con entrevistas individuales de los sujetos.

Creemos que este trabajo nos ha permitido conocer, utilizando técnicas de representación del conocimiento y de análisis del contenido de las historias profesionales de los participantes, cuáles son las preocupaciones del personal de enfermería en relación a su propia formación.

En conclusión, la formación del personal de enfermería aparece fuertemente vinculada a la ayuda de otros profesionales no preparados para la docencia. Sería muy importante aportar a esta faceta de la formación un carácter más reglado científicamente, lo que supondría, además, la adaptación de metodologías de enseñanza, ajustándolas al contexto laboral.

\section{Bibliografía}

1. Schvaneveldt RW, ed. Pathfinder Associative Networks. Studies in knowledge organization. Norwood: Ablex; 1989. 
2. Piera AM. Análisis de la formación de las enfermeras. I. De los usos y métodos de investigación cualitativa. Cultura de los Cuidados 1998; 2: 46-51.

3. Cuesta C. Investigación cualitativa en el campo de la salud. Rol de Enfermería 1997; 232.

4. Bull MJ. Using qualitative methods in teaching undergraduate students research. Nursing \& Healthcare 1992; 13: 378-81.

5. García do Nascimento JM. Construcción y validación de una escala de percepción personal de competencias profesionales del prelicenciado en enfermería [tesis doctoral]. Badajoz: Universidad de Extremadura; 2005.

6. Medina JL. La pedagogía del cuidado: racionalidad, tradición y poder en el currículum de enfermería: un estudio interpretativo [tesis doctoral]. Barcelona: Universitat de Barcelona; 1995.

7. Amaya F, Guirao J, Huertas A, Moreno P. Utilidad atribuida por los alumnos de enfermería de primer curso a las prácticas. Educare21 2004; 13.

8. Martínez JM. Vocación en enfermería: entre la etnografía y la cuantificación. Una visión a través de estudiantes de enfermería. Cádiz: Colegio Oficial de Enfermería de Cádiz; 2004.

9. Pérez M, Cruz L, Rojas M. Aproximación fenomenológica a las experiencias de aprendizaje clínico de los estudiantes de enfermería. Enfermeras 2001; 38: 19-24.
10. Duro JC. El discurso de los profesionales de atención primaria de la Comunidad de Madrid acerca del trabajo con grupos: sobre técnicas y técnicos. Revista Española de Salud Pública 2003; 77: 615-27.

11. Pérez C, Alameda A, Albéniz C. La formación práctica en enfermería en la escuela universitaria de enfermería de la comunidad de Madrid. Opinión de los alumnos y de los profesionales asistenciales. Un estudio cualitativo con grupos de discusión. Revista Española de Salud Pública 2002; 76: 517-53.

12. Mitchell TH. Present tense commentary: a qualitative research technique. Nurse Education Today 1999; 19: 151-8.

13. Osgood CE. The representation model and relevant research methods. In De Sola-Pool I, ed. Trends in content analysis. Urbana, IL: University of Illinois Press; 1959.

14. KNOT Software. Interlink. URL: http://interlinkinc.net.

15. Casas L, Luengo R. Representación del conocimiento y aprendizaje: teoría de los conceptos nucleares. Revista Española de Pedagogía 2004; 227: 59-84.

16. Casas L. El estudio de la estructura cognitiva de alumnos a través de las Redes Asociativas Pathfinder. Aplicaciones y posibilidades en geometría. In: Premios Nacionales de Investigación e Innovación Educativa 2003. Madrid: Ministerio de Educación y Ciencia, CIDE; 2005. p. 573-607. 